\title{
Taking a Closer Look at Methodological Quality: JACM Partners with Cochrane Complementary Medicine
}

\author{
Holger Cramer $^{1}$ and L. Susan Wieland ${ }^{2}$
}

Rigor and Reproducibility

$\mathbf{T}$ Wo CORNERSTONES OF SCIENTIFIC advancement are methodological rigor and reproducibility of scientific findings. Although it may not seem immediately obvious, these two pillars are highly interdependent. ${ }^{1}$

According to the US National Institutes of Health, methodological rigor in design and realization of scientific studies is "the strict application of the scientific method to ensure robust and unbiased experimental design, methodology, analysis, interpretation and reporting of results.", Lack of methodological rigor can have many causes: fortunately, probably the rarest cause is deliberate scientific misconduct, i.e. the purposeful production or falsification of data. However, in times of increased pressure to publish and increasing competition for research funds, the importance of scientific misconduct should not be underestimated, as it is the reason for more than two thirds of the retractions of scientific papers. Since 1975, the number of retractions due to scientific misconduct has increased tenfold (with the USA and Germany clearly taking the top positions in retractions because of fraud). ${ }^{3}$ Much more dangerous than conscious fraud, however, because it is more blurred and often not recognized as such even by the scientists concerned, is an unconscious lack of scientific rigor: due to a lack of methodological training or to cognitive bias, cherry-picking is practiced, and results are interpreted or selected in conformity to a preferred hypothesis. Or the work is simply not done precisely and conscientiously enough - either out of inexperience or out of carelessness, precisely because experience teaches that it almost always works out well. But only almost always. ${ }^{4}$

The consequences of lack of scientific rigor, whether deliberate or negligent, are manifold and, especially in clinical research, are a potential threat to public health if the implementation of a therapeutic method is based on biased efficacy and safety data.

In addition, however, lack of rigor is also one of the most important threats to reproducibility. Replication of research findings is a basic requirement for making generally applicable treatment recommendations. Without independent replications, individual research results cannot be detached from their specific context; they only allow statements related to the location and time, where and when they were obtained. And, in health research, related to the respective therapists and patients. ${ }^{5}$ Unfortunately, the independent replication of study results, although essential for therapy decisions, is valued much less than supposedly groundbreaking new findings. What replication would ever have made it to the front pages of major newspapers? And grants also are much more likely to be awarded for "new ideas" than for replications. And so this valuable scientific tool is underutilized. ${ }^{5,6}$ However, if these supposed breakthrough results are the consequence of a lack of methodological rigor, then an attempt at replication is essential, as it can refute the biased results. If it is lacking, this leads to wrong decisions - and to public health hazards.

There are unfortunately still people for whom traditional, complementary, alternative, and integrative medicine (TCIM) research and methodological rigor is an oxymoron, i.e. a pairing of two mutually exclusive terms. Wikipedia (not a scientific source, of course, but often the first access to medical information for the public) lumps together traditional, complementary, alternative, and integrative medicine, defining them all as "any practice that aims to achieve the healing effects of medicine, but which lacks biological plausibility and is untested, untestable, or proven ineffective" and claiming that "they reside outside medical science, and rely on pseudoscience." 7 In the further explanation it is listed as the main point of difference to conventional medicine that TCIM cannot or will not be tested by means of scientific studies.

\footnotetext{
${ }^{1}$ Department of Internal and Integrative Medicine, Evang. Kliniken Essen-Mitte, Faculty of Medicine, University of Duisburg-Essen, Essen, Germany.

${ }^{2}$ Cochrane Complementary Medicine, Center for Integrative Medicine, University of Maryland School of Medicine, Baltimore, MD, USA.
} 


\section{JACM Partners with Cochrane Complementary Medicine}

From this it becomes clear that methodological rigor is at least as important in TCIM as it is in biomedicine: in the latter it is tacitly assumed, in the former it is just as tacitly denied. Thus, to overcome this prejudice, TCIM studies must be at least as rigorous as conventional medical ones. JACM has always stood for methodological rigor; in the future, we will focus on and communicate it even more explicitly.

An important building block in this methodology initiative is the journal's brand-new partnership with Cochrane Complementary Medicine starting with this issue. Cochrane probably stands for methodological rigor like no other organization. Since Cochrane was founded in 1993 as the Cochrane Collaboration, it has been in the forefront of quality and innovation in systematic reviews and meta-analyses, with regard not only to technical standards but also to standards for transparency and the identification and reduction of methodological bias. Systematic reviews, the comprehensive identification, critical assessment and summarizing of information on a specific research question, and optionally included meta-analyses, the statistical combining of information from separate but similar studies, are an increasingly widely used and trusted method to understand and convey the overall evidence on a particular health question. ${ }^{8}$

Cochrane itself is an international non-profit organization focused on the preparation of high-quality systematic reviews to inform health decision-making, with over 13,000 members and 50,000 supporters from more than 130 countries as of 2018. ${ }^{9}$ The organization consists of Cochrane Review Groups that produce Cochrane reviews, Methods Groups that develop the methods for Cochrane reviews, Geographic Groups that provide a hub for Cochrane activities in different geographic areas, and Fields that support Cochrane in topic areas that cross multiple health conditions. The main product of Cochrane is the Cochrane Library, an online repository of databases the most relevant of which is the Cochrane Database of Systematic Reviews, a continuously updated database containing the full text of all Cochrane systematic reviews and Cochrane protocols (i.e., pre-specified procedures) for systematic reviews.

The Cochrane Complementary Medicine Field was founded in 1996, shortly after the founding of the initial Cochrane Review Groups, to support Cochrane efforts in ensuring the completeness, relevance, quality and accessibility of systematic review evidence on TCIM. For example, Cochrane Complementary Medicine promotes the assessment and improvement of methods for controlled trials and systematic reviews in TCIM, facilitates the identification and availability of difficult-to-find controlled trials in TCIM, develops and delivers educational workshops on the preparation and interpretation of Cochrane evidence, and supports the production and dissemination of individual Cochrane reviews in acupuncture, yoga, and other TCIM modalities. ${ }^{10}$ The Field also promotes the dissemination of Cochrane review evidence to interested audiences through multiple venues, including summaries and commentaries in TCIM journals.

This issue marks the introduction of a regular Cochrane Column, consisting of summaries of the most recent Cochrane evidence relevant to our audience. The goal of these synopses of Cochrane reviews is to provide insight into the current focus and findings of Cochrane reviews in TCIM and related whole-person topic areas, together with a link to the Cochrane review for more detailed information. They provide quick and immediate access to the results of relevant reviews. In addition, they serve as a cornerstone in JACM's new methodology initiative: Cochrane reviews not only offer the highest methodological standard of systematic reviews, they also contain particularly sophisticated methods for assessing the quality and risk of bias in the original papers they contain, and for assessing the overall confidence in the review findings. They thus help to separate the wheat from the chaff.

JACM is proud to partner with Cochrane Complementary Medicine to strengthen methodological rigor in the field but also to demonstrate the methodological quality that many TCIM studies now have.

\section{References}

1. National Institutes of Health. Rigor and reproducibility. Online document at: www.nih.gov/research-training/rigorreproducibility, accessed March 24, 2021.

2. National Institutes of Health. Guidance: rigor and reproducibility in grant applications. Online document at: https:// grants.nih.gov/policy/reproducibility/guidance.htm, accessed March 24, 2021.

3. Fang FC, Steen RG, Casadevall A. Misconduct accounts for the majority of retracted scientific publications. Proc Natl Acad Sci USA. 2012;10917028-33.

4. Hofseth LJ. Getting rigorous with scientific rigor. Carcinogenesis. 2018;39:21-25.

5. Schmidt S. Shall we really do it again? The powerful concept of replication is neglected in the social sciences. Rev Gen Psychol. 2009;13:90-100.

6. Ioannidis JPA. Why most clinical research is not useful. PLoS Med. 2016;13:e1002049.

7. Wikipedia. Alternative medicine. Online document at: https://en.wikipedia.org/wiki/Alternative_medicine, accessed March 24, 2021.

8. Ahn E, Kang H. Introduction to systematic review and meta-analysis. Korean J Anesthesiol. 2018;71:103-112.

9. Chandler J, Cumpston M, Thomas J, Higgins JPT, Deeks JJ, Clarke MJ. Chapter I: Introduction. In: Higgins JPT, Thomas J, Chandler J, Cumpston M, Li T, Page MJ, Welch VA, eds. Cochrane Handbook for Systematic Reviews of Interventions version 6.2 (updated February 2021). Cochrane, 2021. Online document at: www.training .cochrane.org/handbook, accessed April 1, 2021.

10. Cochrane Complementary Medicine. Online document at: https://cam.cochrane.org, accessed April 1, 2021.

Address correspondence to: Holger Cramer, PhD Research Director

Department of Internal and Integrative Medicine Evang. Kliniken Essen-Mitte University of Duisburg-Essen Essen Germany

E-mail: H.Cramer@kem-med.com 